\title{
Image-Based Respiratory Motion Compensation for Fluoroscopic Coronary Roadmapping
}

\author{
Ying Zhu*, Yanghai Tsin, Hari Sundar, and Frank Sauer \\ Siemens Corporate Research, \\ 755 College Road East, Princeton, NJ 08540, USA \\ yingzhu@siemens.com
}

\begin{abstract}
We present a new image-based respiratory motion compensation method for coronary roadmapping in fluoroscopic images. A temporal analysis scheme is proposed to identify static structures in the image gradient domain. An extended Lucas-Kanade algorithm involving a weighted sum-of-squared-difference (WSSD) measure is proposed to estimate the soft tissue motion in the presence of static structures. A temporally compositional motion model is used to deal with large image motion incurred by deep breathing. Promising results have been shown in the experiments conducted on clinical data.
\end{abstract}

\section{Introduction}

Motion compensation is an important issue for image guided coronary angioplasty procedures. Besides live fluoroscopic images, imaging techniques such as coronary roadmapping and multimodal image fusion have been introduced to provide clinicians additional image guidance. In coronary roadmapping [12, a dynamic coronary roadmap is obtained from dye-injected images showing vascular structures under cardiac motion. When the contrast medium disappears, the $2 \mathrm{D}$ roadmap can be superimposed on live fluoroscopy to provide immediate feedback in properly directing guidewires or placing stents. Similarly, 3D vessel roadmap extracted from cardiac CTA or MRA can be overlaid on live fluoroscopy [3 477, to provide detailed vessel information such as calcification and tortuosity which are important success factors for percutaneous coronary interventions. In both cases, reliable motion compensation is required to dynamically move $2 \mathrm{D}$ or 3D coronary roadmaps to properly match live fluoroscopy, especially when the contrast medium has disappeared and the coronary structures are not visible from fluroscopic images.

Cardiac and respiratory motion are the main sources of motion observed in coronary fluoroscopic images. The technique of ECG gating is commonly used to deal with cardiac motion, where roadmap images are overlaid on cardiac gated frames. Motion over the respiratory cycle is generally less reproducible and there are a few drawbacks associated with respiratory gating [5]. Different motion models including translation, rigid body and affine transformations

\footnotetext{
^ Corresponding author.
}

T. Jiang et al. (Eds.): MICCAI 2010, Part III, LNCS 6363, pp. 287-294, 2010.

(C) Springer-Verlag Berlin Heidelberg 2010 
as well as statistical models have been investigated by several studies [56/7], to characterize the effect of respiration on the heart and coronary arteries. In contrast to the previous work which rely on $3 \mathrm{D}$ volumetric data or biplane acquisition, we explore a 2D image-based approach and present a new method to estimate the image motion incurred by respiration from monoplane fluoroscopic images. The motion estimates allow dynamic overlay of coronary roadmaps to match live fluoroscopy.

Image-based motion estimation has been explored in the context of digital subtraction angiography 11,12 which dealt mainly with cardiac motion correction. Image-based cardiac and respiratory cycle synchronization techniques have been discussed in 8. Other techniques rely on guidewire tracking 910] to locate a coronary artery in fluoroscopic images. An interesting observation about cardiac fluoroscopy is that regardless of whether the contrast medium, guidewires or other devices are present or not, image motion of the soft tissues of the heart is consistently visible and measurable, which suggests the motion of the coronary arteries especially when the contrast medium is not seen. This motivates us to explore the motion information embedded in the broader image region of the heart, rather than focusing on local anatomical landmarks or devices. In addition, mixed layers of static bone structures and moving soft tissues in cardiac fluoroscopic images pose a challenge in recovering soft tissue motion. Our proposed method of image-based respiratory motion compensation through recovering soft tissue motion is a new idea for coronary roadmapping using monoplane fluoroscopic images. To our knowledge, it is also the first attempt to recover soft tissue motion with a special handling of static structures.

\section{Method}

In X-ray imaging, image intensity is determined by the energy flux which undergoes exponential attenuation through layers of tissues. With logarithmic postprocessing, the intensity can be described as an additive superposition of multiple tissue layers undergoing different movements. Previous work focused on separating transparent layers from one or multiple images 131516, based on the assumption that the layers remain static, or their motion is either known beforehand or irrelevant to layer separation. Jointly recovering layers and their motion from fluoroscopic images remains an open problem.

\subsection{Image-Based Motion Estimation}

For motion compensation, the goal is to extract the coronary motion incurred by respiration. We consider a simplified model with two main layers, a static structure layer including bone tissues and a moving soft tissue layer of the heart including coronary arteries. Denote $I_{t}(\mathbf{x}), I^{s}(\mathbf{x})$ and $I_{t}^{d}(\mathbf{x})$ as the intensity value of pixel $\mathbf{x}$ in the fluoroscopic image, the static layer and the soft tissue layer at time $t$ respectively. They are related through additive superposition.

$$
I_{t}(\mathbf{x})=I^{s}(\mathbf{x})+I_{t}^{d}(\mathbf{x})
$$


In coronary roadmapping, a 2D or 3D coronary roadmap is initially overlaid on one or multiple dye-injected fluoroscopic images through vessel-based registration 4. These fluoroscopic images are referred to as the reference images. Once the contrast medium disappears, we perform motion compensation by recovering soft tissue motion between the reference images and live fluoroscopic images. The recovered motion is used to appropriately place the roadmap in the dye-free live images. Denote $I_{t_{0}}(\mathbf{x})$ as a reference image. An incoming live image $I_{t}$ is related to $I_{t_{0}}$ through soft tissue motion

$$
\begin{gathered}
I_{t_{0}}(\mathbf{x})=I^{s}(\mathbf{x})+I_{t_{0}}^{d}(\mathbf{x}) \\
I_{t}(\mathbf{x})=I^{s}(\mathbf{x})+I_{t_{0}}^{d}(W(\mathbf{x} ; P)) \quad(\mathbf{x} \in \Omega)
\end{gathered}
$$

$W(\mathbf{x} ; P)$ denotes the model charactering soft tissue motion between $t_{0}$ and $t$ with parameters $P$. $\Omega$ denotes the image area of the heart exclusive of the coronary arteries due to contrast disappearance. Our problem is to estimate the soft tissue motion $W(\mathbf{x} ; P)$ from $I_{t_{0}}$ and $I_{t}$.

Without the layer of static structures $I^{s}$, we have $I_{t_{0}}=I_{t_{0}}^{d}, I_{t}=I_{t}^{d}$ and $I_{t}(\mathbf{x})=I_{t_{0}}(W(\mathbf{x} ; P))$. Motion estimation can be solved by the well-known LucasKanade (LK) algorithm [14], which uses a Gauss-Newton gradient descent method to minimize the sum of squared difference (SSD)

$$
S S D(P)=\frac{1}{N_{\Omega, P}} \sum_{\mathbf{x} \in \Omega_{P}^{-1}}\left\|I_{t}(\mathbf{x})-I_{t_{0}}(W(\mathbf{x} ; P))\right\|^{2}
$$

where $\Omega_{P}^{-1}=W^{-1}(\Omega ; P)$ and $N_{\Omega, P}$ is the number of pixels in $\Omega_{P}^{-1}$. The motion parameters are iteratively updated with small increments.

$$
\begin{gathered}
\delta P=H^{-1} \sum_{\mathbf{x} \in \Omega_{P}^{-1}} \nabla I_{t_{0}}^{T}\left(\frac{\partial W}{\partial P}\right)^{T}\left(I_{t}(\mathbf{x})-I_{t_{0}}(W(\mathbf{x} ; P))\right) \\
\left(H=\sum_{\mathbf{x} \in \Omega_{P}^{-1}}\left(\frac{\partial W}{\partial P} \nabla I_{t_{0}}\right)\left(\frac{\partial W}{\partial P} \nabla I_{t_{0}}\right)^{T}\right)
\end{gathered}
$$

This involves the calculation of image gradients $\nabla I_{t_{0}}$ and the Jacobian of the motion transformation function $\frac{\partial W}{\partial P}$.

\subsection{Dealing with Static Structures}

With a layer of static structures such as bone tissues, visible skin markers and devices, the image intensities $I_{t}, I_{t_{0}}$ have an added component $I^{s}$ which remains static over time. Without a proper treatment, the static structures would have an adverse effect on the estimation of soft tissue motion. Here we present a novel approach to deal with static structures in the estimation of soft tissue motion. Note that the motion parameters obtained by (5) are essentially determined by two gradient terms, the spatial image gradient $\nabla I_{t_{0}}$ and the temporal image gradient $I_{t}(\mathbf{x})-I_{t_{0}}(W(\mathbf{x} ; P))$.

$$
\begin{gathered}
\nabla I_{t_{0}}=\nabla I_{t_{0}}^{d}+\nabla I^{s} \\
I_{t}(\mathbf{x})-I_{t_{0}}(W(\mathbf{x} ; P))=I_{t}^{d}(\mathbf{x})-I_{t_{0}}^{d}(W(\mathbf{x} ; P))+I^{s}(\mathbf{x})-I^{s}(W(\mathbf{x} ; P))
\end{gathered}
$$


Ideally the soft tissue motion should be estimated from the dynamic components $\nabla I_{t_{0}}^{d}$ and $I_{t}^{d}(\mathbf{x})-I_{t_{0}}^{d}(W(\mathbf{x} ; P))$, which would require knowing $I^{s}$ exactly to remove $\nabla I^{s}$ and $I^{s}(\mathbf{x})-I^{s}(W(\mathbf{x} ; P))$ from the spatial and temporal gradient terms. Instead of requiring the full knowledge of $I^{s}$, we explore the gradient fields $\nabla I_{t}, \nabla I_{t_{0}}$ to identify local image structures with strong image gradients which are likely to come from static structures. This is motivated by the fact that salient image structures characterized by high gradient values are sparse and distributed locally in $I^{s}$. For the vast homogeneous areas in $I^{s}, \nabla I^{s}$ is negligible, i.e. $\nabla I^{s} \approx 0, I^{s}(W(\mathbf{x} ; P)) \approx I^{s}(\mathbf{x})$ given that the motion is small, thus we have

$$
\begin{aligned}
\nabla I_{t_{0}}(\mathbf{x}) & \approx \nabla I_{t_{0}}^{d}(\mathbf{x}) \\
I_{t}(\mathbf{x})-I_{t_{0}}(W(\mathbf{x} ; P)) & \approx I_{t}^{d}(\mathbf{x})-I_{t_{0}}^{d}(W(\mathbf{x} ; P))
\end{aligned}
$$

This means that if we ignore the local image structures that have high gradient values and remain static across $I_{t}$ and $I_{t_{0}}$, we can obtain an approximate solution for soft tissue motion using $I_{t}$ and $I_{t_{0}}$.

To identify the local static structures with strong gradients, motivated by [16], we compute in the gradient field the local correlation coefficient between images acquired at different times.

$$
\rho(\mathbf{x})=\left.\frac{\operatorname{Cov}\left(\left|\nabla I_{t}\left(\mathbf{x}^{\prime}\right)\right|,\left|\nabla I_{t_{0}}\left(\mathbf{x}^{\prime}\right)\right|\right)}{\sqrt{\left(\operatorname{Var}\left(\left|\nabla I_{t}\left(\mathbf{x}^{\prime}\right)\right|\right) \operatorname{Var}\left(\left|\nabla I_{t_{0}}\left(\mathbf{x}^{\prime}\right)\right|\right)\right)}}\right|_{\mathbf{x}^{\prime} \in \mathcal{N}(\mathbf{x})} \quad(-1 \leq \rho(\mathbf{x}) \leq 1)
$$

$\operatorname{Cov}\left(\left|\nabla I_{t}\right|,\left|\nabla I_{t_{0}}\right|\right)$ and $\operatorname{Var}\left(I_{t / t_{0}}\right)$ are the covariance and variance terms computed in a local neighborhood $\mathcal{N}(\mathbf{x})$. The correlation coefficient $\rho(\mathbf{x})$ is bounded between -1 and 1 . The more consistent the local structures are across images, the higher the correlation coefficient is. $\rho(\mathbf{x})=1$ when the local structures are exactly the same in both images. In practice, when multiple incoming fluoroscopic images or reference images are available, the local correlation coefficient over multiple images is computed as the statistical mean of the local correlation coefficient between every image pair.

Using the local correlation coefficient, we propose to estimate soft tissue motion by minimizing a weighted SSD (WSSD) term defined as follows.

$$
\begin{gathered}
W S S D(P)=\frac{1}{\mathcal{K}} \sum_{x \in \Omega_{P}^{-1}} \kappa(\mathbf{x}) \kappa(W(\mathbf{x} ; P))\left\|I_{t}(\mathbf{x})-I_{t_{0}}(W(\mathbf{x} ; P))\right\|^{2} \\
\kappa(\mathbf{x})=\frac{1}{2}(1-\rho(\mathbf{x})) \quad(0 \leq \kappa(\mathbf{x}) \leq 1) \\
\mathcal{K}=\sum_{x \in \Omega_{P}^{-1}} \kappa(\mathbf{x}) \kappa(W(\mathbf{x} ; P))
\end{gathered}
$$

The weighting function $\kappa(\mathbf{x})$ determines the contribution from each pixel to the estimation of soft tissue motion. For static structures across multiple images, the local correlation coefficient $\rho(\mathbf{x})$ is close to 1 and $\kappa(\mathbf{x})$ is close to 0 , and in the gradient domain their contribution to the WSSD is negligible. Therefore the motion parameters obtained by the WSSD method are determined mainly by the gradient structures of the moving tissues. To solve the WSSD problem, we extend the LK algorithm and iteratively update the motion parameters by

$$
\begin{gathered}
\delta P=\tilde{H}^{-1} \sum_{\mathbf{x} \in \Omega_{P}^{-1}} \nabla I_{t_{0}}^{T}\left(\frac{\partial W}{\partial P}\right)^{T} \kappa(\mathbf{x}) \kappa\left(W(\mathbf{x} ; P)\left(I_{t}(\mathbf{x})-I_{t_{0}}(W(\mathbf{x} ; P))\right)\right. \\
\tilde{H}=\sum_{\mathbf{x} \in \Omega_{P}^{-1}} \kappa(\mathbf{x}) \kappa(W(\mathbf{x} ; P))\left(\frac{\partial W}{\partial P} \nabla I_{t_{0}}\right)\left(\frac{\partial W}{\partial P} \nabla I_{t_{0}}\right)^{T}
\end{gathered}
$$


Note the warped weighting function $\kappa(W(\mathbf{x} ; P))$ suppresses the contribution from the static structures in the warped image $I_{t_{0}}(W(\mathbf{x} ; P))$, similar to what $\kappa(\mathbf{x})$ does to $I_{t}(\mathbf{x})$. Affine motion model is used in our experiments to describe the image motion of the soft tissues incurred by respiration. To improve the motion capture range, we adopt a pyramid implementation to estimate and propagate image motion from coarse to fine resolutions.

Fig. (1) shows how the proposed WSSD-based method works with static structures. We started with a real coronary image as the reference and introduced rotation, scaling and translation to generate a test image. A layer of static grid structures was added to the reference image and the test image through (1). The weighting function computed from the local correlation coefficients well captures the static grid structures. A vesselness map generated by the approach presented in 12 was used to simulate the roadmap overlays. Compared to the standard SSD-based method, the WSSD-based method performs more accurate motion compensation when static structures are present and the roadmap overlay matches the actual coronary arteries more precisely.

\subsection{Compositional Motion Model}

To compensate large image motion incurred by breathing, we introduce a temporally compositional motion model. Assume that the roadmap image is initially overlaid on a reference image $I_{t_{0}}$ acquired at $t_{0}$ and for incoming fluoroscopic images, respiratory motion compensation is carried out on cardiac gated frames. At time $t_{1}$, motion compensation is performed between $I_{t_{1}}$ and $I_{t_{0}}$ to transform the roadmap image to match $I_{t_{1}}$. Denote the motion between $I_{t_{1}}$ and $I_{t_{0}}$ as $W\left(\mathbf{x} ; P_{1,0}\right)$. As the respiratory phase moves away from $t_{0}$, for instance at $t_{2}$, the soft tissue motion observed in the fluoroscopic image can be quite large, which makes direct motion estimation between $I_{t_{2}}$ and $I_{t_{0}}$ difficult. Instead, we first estimate the motion $W\left(\mathbf{x} ; P_{2,1}\right)$ between $I_{t_{2}}$ and $I_{t_{1}}$ assuming the motion between $I_{t_{2}}$ and $I_{t_{1}}$ is much smaller than the motion between $I_{t_{2}}$ and $I_{t_{0}}$. We then use the compositional motion model $W\left(\mathbf{x} ; P_{2,0}\right)=W\left(W\left(\mathbf{x} ; P_{2,1}\right) ; P_{1,0}\right)$ to initialize the motion parameters $P_{2,0}$, and continue with the extended LK algorithm to refine the motion model $W\left(\mathbf{x} ; P_{2,0}\right)$ between $I_{t_{2}}$ and $I_{t_{0}}$. Essentially, $I_{t_{1}}$ serves as an online reference image to relate the live fluoroscopic image $I_{t_{2}}$ to the predetermined reference image $I_{t_{0}}$. In our implementation, multiple online reference images covering a normal respiratory cycle are used in the motion compensation of live fluoroscopic images.

\section{Results}

To test the accuracy of the proposed method for motion compensation, we used fluoroscopic images from 7 clinical cases of chronic total occlusion or stenosis treatment. The data was acquired on Angiographic C-arm systems (AXIOM Artis, Siemens Medical Solution) from different angles. Each image frame has $512 \times 512$ pixels and the pixel size is either $0.216 \mathrm{~mm}$ or $0.308 \mathrm{~mm}$. These cases were chosen because they all had guidewires present throughout the entire image 

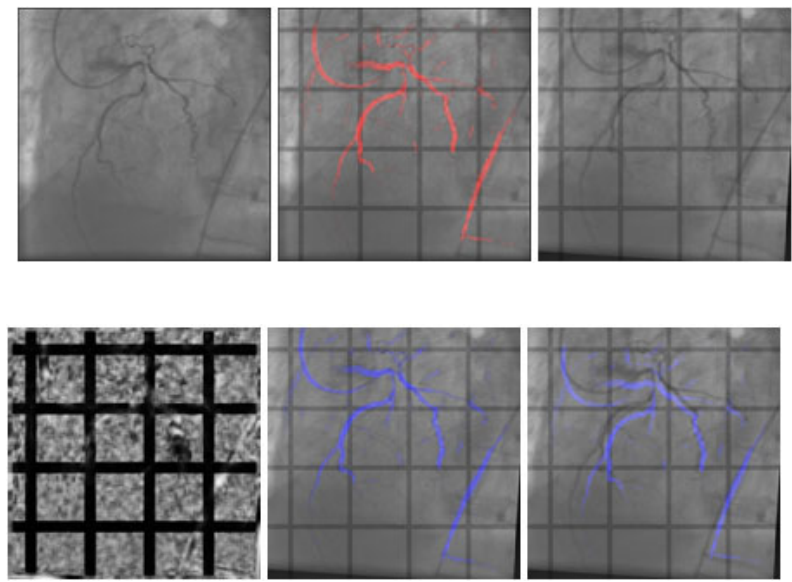

Fig. 1. WSSD-based motion estimation. Top row (left to right): original coronary image; reference image with a vesselness map shown in red simulating a coronary roadmap; test image. Bottom row (left to right): weighting function computed from local correlation coefficients; overlay of vesselness map after WSSD-based motion compensation; overlay of vesselness map after standard SSD-based motion compensation.

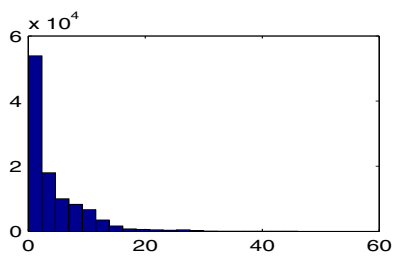

(a)

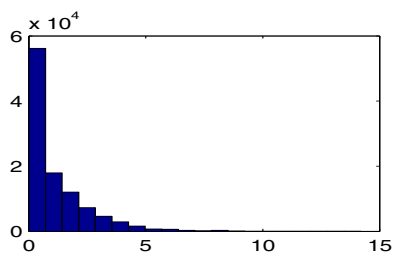

(b)

Fig. 2. Distribution of the misalignment error. (a) Error in pixels. (b) Error in millimeters.

sequences, which provided the ground truth of vessel centerlines for evaluation. Images with dye injection or visible guidewires were used as the reference images for the initial roadmap overlay. In each reference image, we manually labeled the centerline or the guidewire of one coronary artery to simulate the initial roadmap overlay. We performed motion compensation on a total of 106 frames, and used the estimated motion parameters to transform the initial roadmaps to match the test images. In each test image, we manually labeled the guidewire and used it as the ground truth for the coronary centerline. We then compared the ground truth of the coronary centerlines with the motion compensated roadmap overlays. As a misalignment measure, the distances between the motion compensated roadmap and the ground truth labels were calculated.

Since the fluoroscopic sequences used in testing were captured by long focal length perspective models, i.e., near orthographic projection of the 3D volume, pixel spacing in 2D images can be mapped to the distance between corresponding points in the $3 \mathrm{D}$ space through scaling by a factor. This is valid in the 

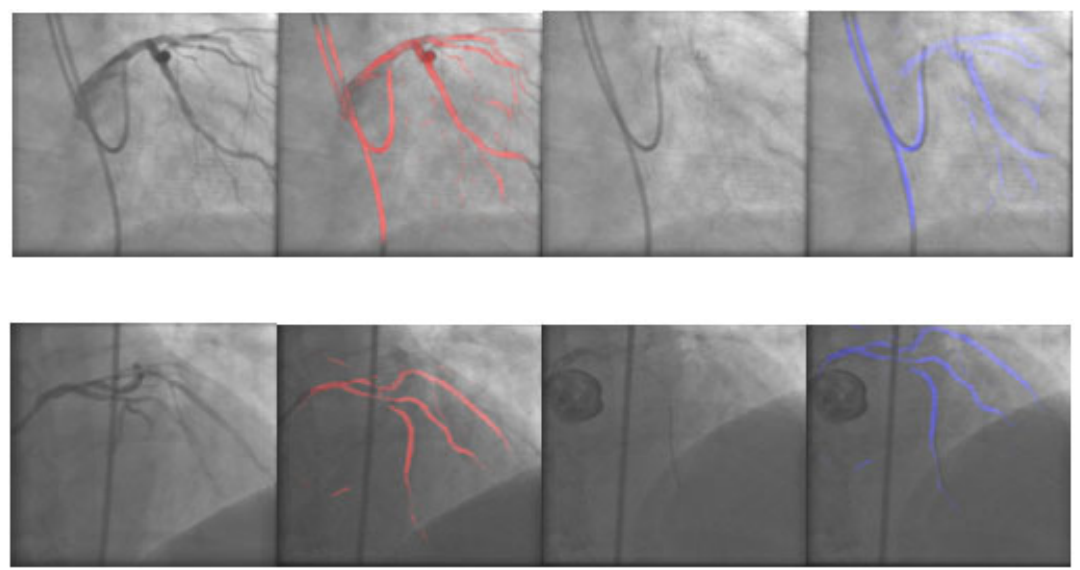

Fig. 3. Coronary roadmapping shown in pairs of an original image and the image with roadmap overlay. Roadmaps in reference images are shown in red. Motion compensated roadmaps in test images are shown in blue.

plane orthogonal to the viewing direction, regardless of the $3 \mathrm{D}$ geometry of the imaged subject or its depth. Fig. 2 shows the distribution of the misalignment error in pixels and in millimeters. The mean, median and standard deviation of the misalignment are 4.1064, 2.2361 and 4.9317 pixels, or $1.1443,0.6469$ and $1.3801 \mathrm{~mm}$ respectively. The two error distributions are slightly different because of the different pixel sizes in the testing sequences. A few examples of motion compensated roadmap overlays are shown in Fig. 3.

\section{Discussions}

We have presented a novel image-based method of respiratory motion compensation for coronary roadmapping in fluoroscopic images. An extended LK algorithm based on weighted SSD is proposed to robustly estimate soft tissue motion when layers of static structures are present. For coronary roadmapping, the coronary locations are essentially inferred from the soft tissue motion when the contrast medium is not present. Even though a simplified two-layered compositional model and a global affine motion model are used to characterize the content and the dynamics of the fluoroscopic images of the heart over respiratory cycles, the initial results on real clinical data look very promising. Future directions include investigation of more elaborated models including local motion models to better characterize soft tissue motion incurred by respiration.

\section{References}

1. Tobis, J., Johnston, W., Montelli, S., Henderson, E., Roeck, W., Bauer, B., Nalcioglu, O., Henry, W.: Digital coronary roadmapping as an aid for performing coronary angioplasty. Am. J. Cardiol. 56(4), 237-241 (1985) 
2. Elion, J.L.: Dynamic coronary roadmapping. United States Patent 4878115, October 31 (1989)

3. A 3D CT Vessel Roadmap Over Live Fluoroscopy for Chronic Total Occlusion, Cath Lab Digest (February 2008), http://www.cathlabdigest.com

4. Ruijters, D., Romeny, B.M., ter, H., Suetens, P.: Vesselness-based 2D-3D registration of the coronary arteries. Int. J. Computer Assisted Radiology and Surgery 4(4), 391-397 (2009)

5. McLeish, K., Hill, D.L.G., Atkinson, D., Blackall, J.M., Razavi, R.: A study of the motion and deformation of the heart due to respiration. IEEE Trans. Medical Imaging 21(9), 1142-1150 (2002)

6. Shechter, G., Ozturk, C., Resar, J.R., McVeigh, E.R.: Respiratory Motion of the Heart From Free Breathing Coronary Angiograms. IEEE Trans. Medical Imaging 23(8), 1046-1056 (2004)

7. King, A.P., Boubertakh, R., Rhode, K.S., Ma, Y.L., Chinchapatnam, P., Gao, G., Tangcharoen, T., Ginks, M., Cooklin, M., Gill, J.S., Hawkes, D.J., Razavi, R.S., Schaeffter, T.: A subject-specific technique for respiratory motion correction in image-guided cardiac catheterisation procedures. Med. Image Anal. 13(3), 419431 (2009)

8. Sundar, H., Khamene, A., Yatziv, L., Xu, C.: Automatic image-based cardiac and respiratory cycle synchronization and gating of image sequences. In: Yang, G.-Z., Hawkes, D., Rueckert, D., Noble, A., Taylor, C. (eds.) MICCAI 2009, Part II. LNCS, vol. 5762, pp. 381-388. Springer, Heidelberg (2009)

9. Baert, S.A.M., Viergever, M.A., Niessen, W.J.: Guide-wire tracking during endovascular intervention. IEEE Trans. on Medical Imaging 22(8), 965-972 (2003)

10. Wang, P., Zhu, Y., Zhang, W., Chen, T., Durlak, P., Bill, U., Comaniciu, D.: Hierarchical guidewire tracking in fluoroscopic sequences. In: SPIE Medical Imaging, vol. 7258, pp. 72591L-72591L-8 (2009)

11. Meijering, E.H.W., Niessen, W.J., Viergever, M.A.: Retrospective Motion Correction in Digital Subtraction Angiography: A Review. IEEE Trans. Medical Imaging 18(1), 2-21 (1999)

12. Zhu, Y., Prummer, S., Chen, T., Ostermeier, M., Comaniciu, D.: Coronary DSA: Enhancing Coronary Tree Visibility through Discriminative Learning and Robust Motion Estimation. In: SPIE Medical Imaging, vol. 7258, pp. 72591L-72591L-8 (2009)

13. Zhang, W., Ling, H., Prummer, S., Zhou, K.S., Ostermeier, M., Comaniciu, D.: Coronary tree extraction using motion layer separation. In: Yang, G.-Z., Hawkes, D., Rueckert, D., Noble, A., Taylor, C. (eds.) MICCAI 2009, Part I. LNCS, vol. 5761, pp. 116-123. Springer, Heidelberg (2009)

14. Baker, S., Matthews, I.: Lucas-Kanade 20 Years On: A Unifying Framework. Int. J. Computer Vision 56(3), 221-255 (2004)

15. Chen, Y., Chang, T.-C., Zhou, C., Fang, T.: Gradient domain layer separation under independent motion. In: ICCV, pp. 694-701 (2009)

16. Sarel, B., Irani, M.: Separating transparent layers through layer information exchange. In: Pajdla, T., Matas, J(G.) (eds.) ECCV 2004. LNCS, vol. 3024, pp. 328-341. Springer, Heidelberg (2004) 